\title{
Matriptase-mediated cleavage of EpCAM destabilizes claudins and dysregulates intestinal epithelial homeostasis
}

\author{
Chuan-Jin Wu, Xu Feng, Michael Lu, Sohshi Morimura, and Mark C. Udey
}

Dermatology Branch, Center for Cancer Research, National Cancer Institute, NIH, Bethesda, Maryland, USA.

\begin{abstract}
Congenital tufting enteropathy (CTE) is a severe autosomal recessive human diarrheal disorder with characteristic intestinal epithelial dysplasia. CTE can be caused by mutations in genes encoding EpCAM, a putative adhesion molecule, and HAI-2, a cell surface protease inhibitor. A similar phenotype occurs in mice whose intestinal epithelial cells (IECs) fail to express the tight junction-associated protein claudin-7. EpCAM stabilizes claudin-7 in IECs, and HAI-2 regulates the cell surface serine protease matriptase, a known modifier of intestinal epithelial physiology. Therefore, we hypothesized that HAI-2, matriptase, EpCAM, and claudin-7 were functionally linked. Herein we have demonstrated that active matriptase cleaves EpCAM after Arg80 and that loss of HAI-2 in IECs led to unrestrained matriptase activity and efficient cleavage of EpCAM. Cleavage of EpCAM decreased its ability to associate with claudin-7 and targeted it for internalization and lysosomal degradation in conjunction with claudin-7. CTE-associated HAI-2 mutant proteins exhibited reduced ability to inhibit matriptase and also failed to efficiently stabilize claudin-7 in IECs. These results identify EpCAM as a substrate of matriptase and link HAI-2, matriptase, EpCAM, and claudin-7 in a functionally important pathway that causes disease when it is dysregulated.
\end{abstract}

\section{Introduction}

Truncating and selected missense mutations in EPCAM (encoding epithelial cell adhesion molecule [EpCAM; CD326]) cause a severe autosomal recessive childhood diarrheal syndrome termed congenital tufting enteropathy (CTE) $(1,2)$. CTE is characterized by widespread small intestinal epithelial dysplasia, and intestinal mucosal biopsies demonstrate distinctive "tufts" of epithelial cells at the tips of blunted villi $(1,3)$. EpCAM is a cell surface glycoprotein that is present in many developing epithelia, some adult epithelia (including intestine), carcinomas, tumor-initiating cells, circulating tumor cells, and tissue and embryonic stem cells $(4,5)$. Although EpCAM was initially reported to mediate intercellular adhesion directly via homotypic interactions (6), subsequent studies have suggested that EpCAM modulates epithelial cell physiology via several seemingly nonoverlapping mechanisms (7-9). Definitive insights into EpCAM function may come from studies of patients and mice with mutant EPCAM alleles. Despite the wide tissue distribution of EpCAM, patients with CTE do not exhibit prominent extraintestinal features (1). Mice with germline null mutations in Epcam develop the murine equivalent of CTE and die within 2 weeks after birth (10, 11). Consistent with EpCAM's claudin-stabilizing effects (12), intestinal expression of selected claudins, including claudin-7, is markedly decreased in mice and humans with EPCAM mutations (3, 10). The strong similarities between the phenotypes of Epcam and $C l d n 7$ knockout mice suggest that EpCAM-claudin interactions are extremely important in the intestine $(8,13,14)$.

Conflict of interest: The authors have declared that no conflict of interest exists Submitted: May 5, 2016; Accepted: November 22, 2016.

Reference information: J Clin Invest. 2017;127(2):623-634

https://doi.org/10.1172/JCl88428.
Recent studies of CTE patients revealed that a significant minority of individuals harbor mutations in SPINT2 and not in EPCAM (2). SPINT2 encodes a cell membrane-associated Kunitz type 2 serine protease inhibitor, HAI-2, that can regulate the activity of a variety of proteases (15). The cell surface serine protease matriptase is among the enzymes that can be inhibited by HAI-2 indirectly, and possibly directly $(15,16)$. Matriptase is produced as a zymogen, and it becomes fully active only after processing by prostasin, another membrane-associated serine protease, or by matriptase itself (16-18). Both HAI-2 and the closely related protease inhibitor HAI-1 are regulators of the proteolytic cascade that includes prostasin and matriptase $(16,19,20)$. Matriptase influences tight junction composition and regulates intestinal epithelial cell (IEC) monolayer permeability in vitro (21) and in vivo (22), and loss of matriptase in IECs promotes intestinal carcinogenesis in vivo (23, 24). However, detailed mechanisms by which matriptase regulates intestinal epithelial physiology have not been elucidated, and it is not certain that previously identified matriptase substrates (urokinase plasminogen activator [uPA], EGF receptor, protease-activated receptor-2 [PAR2], and HGF/scatter factor) are involved $(21,25)$.

We hypothesized that there might be a direct link between SPINT2 (HAI-2), matriptase, EpCAM, and claudin-7 that relates to IEC homeostasis and CTE. In the present study, we demonstrate that EpCAM is a physiologically relevant substrate of matriptase. We also determined that loss of HAI-2 in IECs results in matriptase activation that in turn leads to efficient but limited proteolysis of EpCAM at cell surfaces followed by lysosomal degradation of both EpCAM and claudin-7. This pathway is an important determinant of intestinal tissue and cell homeostasis, and it provides a framework for understanding why mutations in any of 3 genes (SPINT2, $E p C A M$, and $C l d n 7)$ can cause CTE in humans and/or in mice. 
A

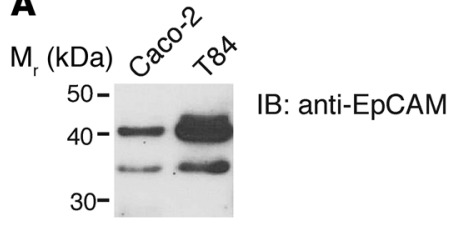

$\mathrm{N}$-terminal sequence of band CE2 $X$ L LPEGAL CE2 R8ORAKPEGAL hEpCAM
B

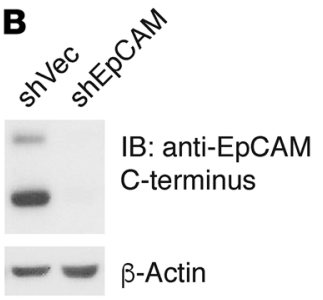

Caco-2
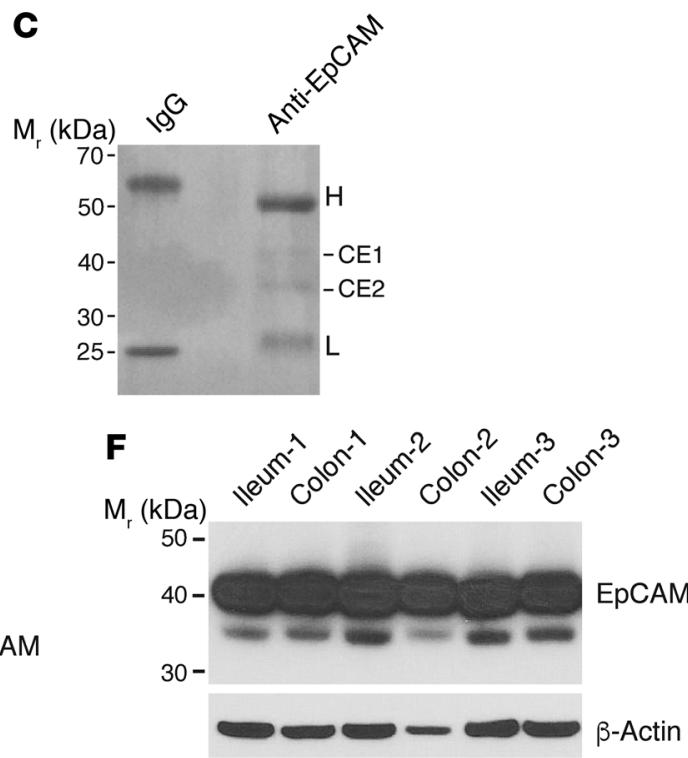

$\beta$-Actin
E

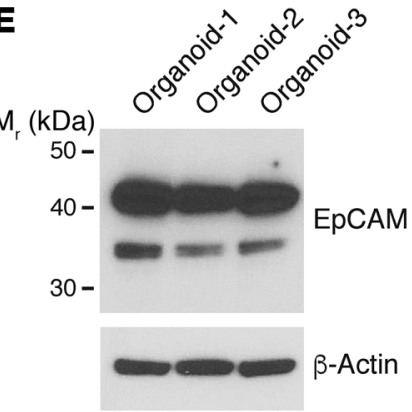

Figure 1. Multiple EpCAM species in IEC lysates reflect proteolytic processing. (A) Nonionic detergent lysates of Caco-2 and T84 cells were resolved via SDSPACE and immunoblotted with polyclonal Ab raised against the entire EpCAM extracellular domain. Representative data from 1 of more than 5 experiments are shown. (B) Stable vector- or EpCAM shRNA-transduced Caco-2 cells were lysed, and SDS-PACE-resolved proteins were immunoblotted with antiEpCAM polyclonal Ab raised against a C-terminal peptide. Representative data from 1 of 3 experiments are shown. (C) RIPA buffer lysates of Caco-2 cells were immunoprecipitated with control IgG or anti-EpCAM mAb. Immunoprecipitates were fractionated using SDS-PAGE and stained with Coomassie blue. Representative data from 1 of 3 experiments are shown. Two EpCAM-reactive species (CE1 and CE2) were recovered, and N-terminal amino acid sequences were determined. $\mathrm{H}$ and $\mathrm{L}$ correspond to IgC heavy and light chains, respectively. (D) $X$ represents an indeterminate amino acid; $A / L$ indicates that both $A$ and $L$ were detected at this position. (E and $\mathbf{F})$ RIPA lysates of small intestine organoids (E) and ileum and colon tissues (F) from three 8- to 10-week-old mice (C57BL/6; Charles River Laboratories) were resolved using reduced SDS-PACE and immunoblotted with the anti-EpCAM polyclonal Ab used in $\mathbf{A}$.

\section{Results}

EpCAM undergoes limited proteolysis in IECs in vitro and in vivo. Anti-EpCAM immunoblots of lysates from human IEC cell lines regularly demonstrated doublets composed of $42-\mathrm{kDa}$ and $36-\mathrm{kDa}$ species when affinity-purified rabbit polyclonal antibodies that react with multiple epitopes in the extracellular domain (Figure 1A) or epitopes contained within a C-terminal peptide (Figure 1B) were used. Although EpCAM heterogeneity could reflect differential glycosylation, we hypothesized that the smaller species represented a $36-\mathrm{kDa}$ proteolytic C-terminal fragment derived from full-length EpCAM as previously proposed by Thampoe et al. (26). To test this, we carried out preparative immunoprecipitation of EpCAM from Caco-2 cell lysates, resolved immunoprecipitated proteins via gel electrophoresis, and attempted N-terminal sequencing of isolated $42-\mathrm{kDa}$ (CE1) and 36-kDa (CE2) species (Figure 1C). We did not obtain useful sequence information from CE1, perhaps because of a blocked N-terminus (27). However, analysis of CE2 resulted in a peptide sequence corresponding to human EpCAM beginning with Arg81 (Figure 1D). To begin to assess the physiologic relevance of this finding, we sought evidence of EpCAM cleavage in IEC organoids and in vivo. Although the predominant bands in immunoblots from organoids and tissues corresponded to full-length EpCAM, we consistently detected small amounts of 36-kDa EpCAM fragments in lysates of murine small IEC organoids (Figure 1E) as well as in lysates of ileum and colon tissues (Figure 1F) from 3 of 3 normal adult mice.
EpCAM associates with, and is a substrate of, the cell surface protease matriptase. Cleavage of EpCAM between Arg80 and Arg81 suggested the action of a trypsin-like serine protease (28). Matriptase is a cell surface serine protease that has been implicated in the regulation of epithelial cell physiology in several tissues, including the gastrointestinal tract $(22,29)$, and we postulated that EpCAM might be a matriptase substrate. Confocal immunofluorescence microscopy revealed that EpCAM and matriptase colocalized at lateral intercellular interfaces in Caco-2 and T84 monolayers (Figure 2, A and B, and Supplemental Figure 1, A-C; supplemental material available online with this article; doi:10.1172/ JCI88428DS1) and in vertical sections of normal human ileum as well (Figure 2C). EpCAM, preferentially uncleaved EpCAM, also coimmunoprecipitated with matriptase when the proteins were coexpressed in HEK293 cells (Supplemental Figure 1D). Furthermore, endogenous matriptase coimmunoprecipitated with EpCAM from lysates of Caco-2 cells that had been treated with the bifunctional cross-linking reagent dithiobis(succinimidyl propionate) (DSP) before lysis (Figure 2D). Coimmunoprecipitation of matriptase and EpCAM from lysates of Caco-2 cells that had not been treated with DSP was inconsistent (data not shown).

The ability of matriptase to cleave EpCAM was tested directly by incubation of recombinant human matriptase catalytic domain with a fusion protein composed of the extracellular N-terminal portion of murine EpCAM and the Fc region of human IgG1 (EpCAM-Ig). Treatment of 61-kDa EpCAM-Ig with matriptase resulted in nearly quantitative conversion to a 55-kDa species (Figure 3, A and B), while 
A

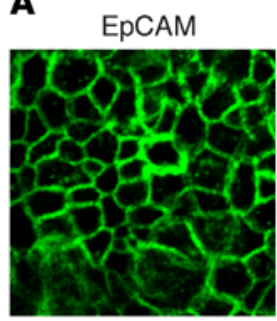

C

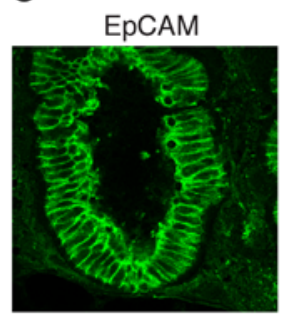

Matriptase

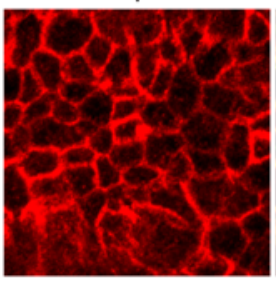

T84

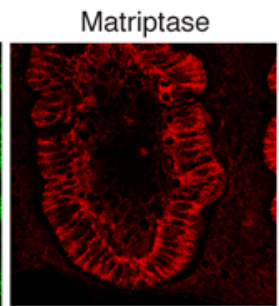

Human ileum
B
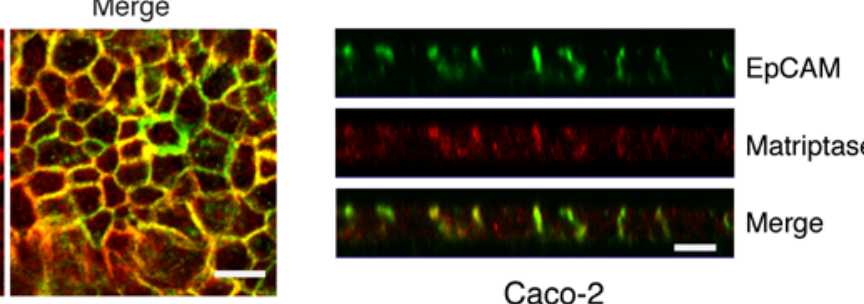

Caco-2

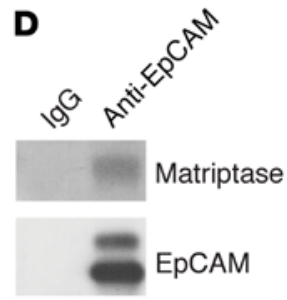

Figure 2. Matriptase colocalizes and physically interacts with EpCAM in IECs. (A and B) Monolayers of T84 (A) or Caco-2 cells (B) were cultured in Transwells and stained for EpCAM (green) and matriptase (red). En face (A) and XZ (B) images from confocal immunofluorescence microscopy are shown. Scale bars: $10 \mu \mathrm{m}$. (C) Frozen sections of human small intestine were stained with anti-EpCAM (green) and anti-matriptase (red) and analyzed via confocal microscopy. Scale bar: $20 \mu \mathrm{m}$. (D) Caco-2 cells were treated with cleavable cross-linking reagent (1 mM DSP) at room temperature for 30 minutes and lysed in $1 \%$ Triton X-100 lysis buffer. Cell lysates were immunoprecipitated with control IgG or anti-EpCAM mAb, and immunoprecipitates were resolved using SDS-PAGE under reducing conditions and immunoblotted with anti-matriptase and anti-EpCAM as indicated. Representative data from 1 of 3 experiments are shown in $\mathbf{A}-\mathbf{D}$.

the Ala30-Gln289 catalytically active fragment of prostasin, another cell surface protease implicated in intestinal epithelial physiology, was without effect, even at 200-fold higher concentrations (Figure 3B). N-terminal amino acid sequencing of the purified $55-\mathrm{kDa}$ fragment (RE2, Figure 3C) confirmed that it corresponded to murine EpCAM-Ig that had been cleaved between Arg80 and Arg81 (Figure 3D). Failure to obtain sequence information from full-length RE1 again suggested the possibility of a blocked N-terminus. The ability of matriptase to initiate EpCAM cleavage in intact cells was initially assessed by cotransfection of HEK293 cells with fixed amounts of human EpCAM expression plasmid and varying amounts of human matriptase expression plasmids (Figure 4A). Whereas only 42-kDa full-length EpCAM was detected in 293 cells transfected with EpCAM expression plasmid alone, introduction of increasing amounts of matriptase expression plasmid led to accumulation of increasing amounts of the 36-kDa EpCAM fragment.

The occurrence of EpCAM cleavage by matriptase in IECs was tested by modulating levels of matriptase in Caco- 2 and T84 cells with siRNAs. Efficient downregulation of matriptase by siRNA-1 led to complete inhibition of EpCAM cleavage (Figure 4, B and C), while siRNA-2 reduced matriptase expression and EpCAM cleavage less effectively. Strikingly, inhibition of EpCAM cleavage in IECs with matriptase siRNAs led to parallel increases in claudin-7 levels (Figure 4C and Supplemental Figure 2).

Cleavage of EpCAM by matriptase in IECs causes dissociation of EpCAM from claudin-7 and subsequent internalization and degradation in lysosomes. We have previously demonstrated that EpCAM associates with claudin-7 and that, in IECs, the expression of EpCAM prevents constitutive lysosomal degradation of claudin-7 (12). We hypothesized that limited proteolysis of EpCAM by matriptase might be linked to cell surface expression of EpCAM, association of EpCAM with claudin-7, and/or lysosomal degradation of EpCAM and/or claudin-7. Indeed, treatment of Caco-2 cells with the lysosomal enzyme inhibitor chloroquine for 20 hours resulted in preferential accumulation of the $36-\mathrm{kDa}$ EpCAM fragment (Figure 5A), suggesting that matriptase-cleaved EpCAM was preferentially targeted for lysosomal degradation.

To determine whether there was a relationship between surface expression of EpCAM and matriptase cleavage, Caco- 2 cells were labeled with the cell-impermeable biotinylating reagent sulfo-NHS-SS-biotin at $4^{\circ} \mathrm{C}$ and warmed to $37^{\circ} \mathrm{C}$ for varying periods, and biotin-modified proteins that had been internalized were enriched and characterized by immunoblotting with relevant antibodies. Proteins that had been internalized were distinguishable from proteins that remained on cell surfaces because biotin residues on the latter were accessible and removable via treatment with the cell-impermeable reducing reagent MESNA. Treatment of Caco-2 cells with sulfo-NHS-SS-biotin at $4^{\circ} \mathrm{C}$ resulted in efficient labeling of $42-\mathrm{kDa}$ and $36-\mathrm{kDa}$ EpCAM, and biotin labeling was completely reversed via immediate MESNA treatment (Figure 5B), suggesting that both EpCAM species were present on IEC surfaces. Incubation of biotin-labeled Caco- 2 cells at $37^{\circ} \mathrm{C}$ resulted in selective internalization of $36-\mathrm{kDa}$ EpCAM, albeit at a somewhat slower rate than the transferrin receptor (Figure $5 \mathrm{~B}$ ).

The potential relationship between EpCAM/claudin-7 association and matriptase-mediated cleavage of EpCAM was tested by assessment of the ability of $42-\mathrm{kDa}$ and $36-\mathrm{kDa}$ EpCAM to coimmunoprecipitate with claudin-7. Claudin-7 was immunoprecipitated from Caco-2-nonionic detergent cell lysates from chloroquine-treated and untreated cells, and EpCAM was detected by 


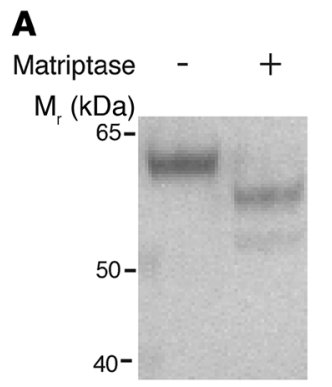

B

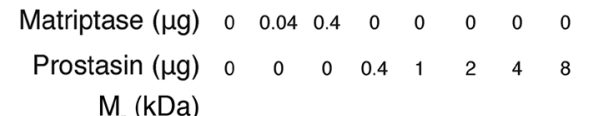
$\mathrm{M}_{\mathrm{r}}(\mathrm{kDa})$

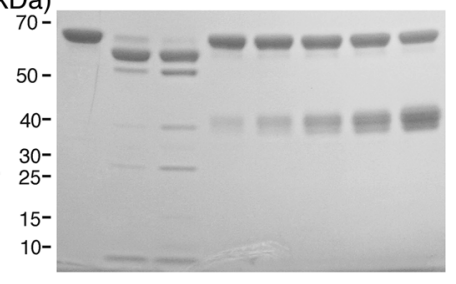

C

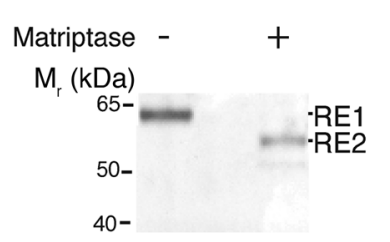

Figure 3. Matriptase cleaves EpCAM. (A) Recombinant murine EpCAM-Ig was incubated with or without recombinant matriptase in "matriptase reaction buffer" at $37^{\circ} \mathrm{C}$ for 1 hour. Reactants were then resolved using SDS-PACE and stained with Coomassie blue. (B) EpCAM-Ig was incubated with indicated amounts of recombinant matriptase or recombinant prostasin in "prostasin reaction buffer" at $37^{\circ} \mathrm{C}$ for 1 hour. Reactants were resolved via SDS-PAGE and stained with Coomassie blue. (C) Recombinant EpCAM-Ig and recombinant matriptase were coincubated as in $\mathbf{A}$, and residual proteins were resolved using SDS-PACE, transferred onto a PVDF membrane, and stained with Coomassie blue. Representative data from 1 of 3 experiments are shown in A-C. (D) The $\mathrm{N}$-terminal amino acid sequence of eluted RE2 was determined, and alignment of the N-terminal sequence of RE2 with that of murine EpCAM is depicted. $\mathrm{X}$ indicates an indeterminate amino acid. immunoblotting. Claudin-7 immunoprecipitates contained only full-length 42-kDa EpCAM even though cell lysates contained abundant amounts of EpCAM 36-kDa fragments (Figure 5C).

The cell surface protease inhibitor HAI-2 is a negative regulator of matriptase-mediated EPCAM cleavage and stabilizes claudin-7 in IECs. Matriptase is synthesized as a zymogen that undergoes a spontaneous single internal cleavage as it is inserted into ER membranes and traffics to cell surfaces (18). Additional proteolysis that results in release of matriptase extracellular domains from cell surfaces is required for complete activation of the enzyme $(16,18)$. The membrane protease prostasin and the membrane-bound serine protease inhibitors HAI- 1 and HAI-2 have been implicated as an activator and as inhibitors, respectively, of the proteolytic cascade that includes matriptase $(16,18)$. Identification of mutations in SPINT2 (the gene encoding HAI-2) in patients with CTE, a disease also caused by EPCAM mutations $(1,2,30)$, led us to speculate that HAI-2 might modulate matriptase-mediated EpCAM proteolysis.

Transfection of 293 cells with increasing amounts of plasmids encoding human HAI-2 in conjunction with fixed amounts of plasmids encoding EpCAM and matriptase resulted in a dosedependent inhibition of EpCAM cleavage (Figure 6A). Introduction of HAI-2 also led to increased cell-associated matriptase, consistent with inhibition of matriptase activation by HAI-2. The possible involvement of HAI-2 as a regulator of matriptasemediated EpCAM cleavage in IECs was then assessed using siRNAs. Introduction of several SPINT2 siRNAs into Caco-2 cells efficiently reduced HAI-2 expression with a corresponding decrease in full-length EpCAM and increased accumulation of $36-\mathrm{kDa}$ EpCAM fragments (Figure 6B). The observed decrease in cellassociated matriptase is consistent with enhancement of endogenous matriptase activation in association with HAI-2 knockdown (16). The effects of activation of this proteolytic pathway on levels of tight junction-associated proteins were subsequently studied in Caco-2 cells and T84 cells. In Caco-2 cells (Figure 6C), inhibition of matriptase expression by matriptase siRNA was associated with decreased EpCAM cleavage and increased claudin-7 accumulation, as expected (Figure 6C and Supplemental Figure
3). In contrast, reduction of SPINT2 expression with siRNA led to efficient matriptase activation, manifested as reduced cellassociated matriptase (16) and enhanced EpCAM cleavage in conjunction with almost complete loss of claudin-7. Claudin-1 levels were also decreased secondary to HAI-2 knockdown at late time points (compare Supplemental Figure 3 with Figure 6C), while occludin levels were not altered. Similar effects of SPINT2 siRNAs on EpCAM and claudin-7 expression were obtained in corresponding experiments with T84 cells (Figure 6D).

The involvement of lysosomal degradation in the pathway activated by loss of HAI-2 was confirmed using the lysosomal protease inhibitor chloroquine. Addition of $100 \mu \mathrm{M}$ chloroquine to Caco-2 cells that had been transfected with SPINT2 siRNAs led to increased accumulation of 36-kDa EpCAM and effectively normalized claudin-7 levels (Figure 6E). Chloroquine also increased levels of 36-kDa EpCAM in control siRNA- and matriptase siRNAtreated cells. Occludin levels in Caco-2 cells were not altered in siRNA- and/or chloroquine-treated cells (Figure 6E).

HAI-2 proteins corresponding to those found in patients with CTE are ineffective inhibitors of matriptase-dependent EPCAM cleavage and fail to stabilize claudin-7 in IECs. Both nonsense and missense mutations in SPINT2 have been described in patients with CTE, and it has been demonstrated that missense mutant HAI-2 proteins are less potent inhibitors than control HAI-2 proteins in cellfree in vitro assays of matriptase activity $(2,31)$. To test the prediction that HAI-2 mutant proteins in CTE patients are less effective inhibitors of the HAI-2/matriptase/EpCAM/claudin-7 pathway that we have described herein than control HAI-2 proteins, we carried out several types of experiments.

In initial experiments, varying amounts of plasmids encoding control or mutant SPINT2 were cotransfected into 293 cells with fixed amounts of EpCAM- and matriptase-encoding plasmids. Although plasmids corresponding to SPINT2 Y163C inhibited EpCAM cleavage to some extent at the highest concentration tested, SPINT2 Y163C plasmid (and corresponding HAI-2 protein) was less effective than comparable amounts of control SPINT2 plasmid (and corresponding protein) (Figure 7A). 
A

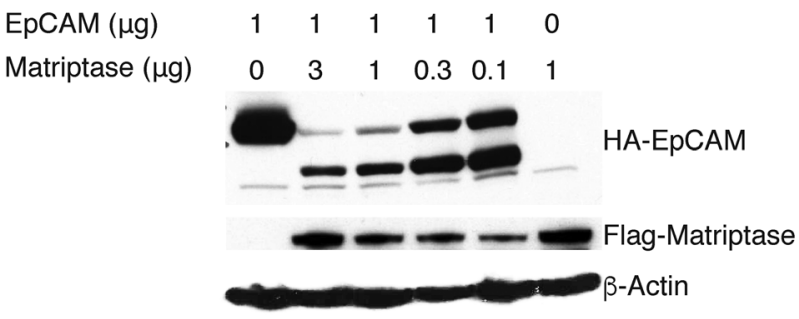

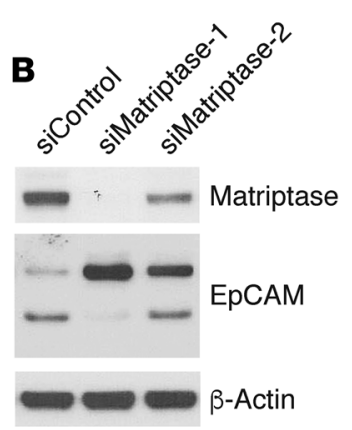

Figure 4. EpCAM is a matriptase substrate in cells, and matriptase regulates claudin-7 levels in IECs. (A) HEK293 cells were transiently transfected with a fixed amount of pcDNA3 encoding EpCAM and varied amounts of matriptase expression plasmid. After 48 hours, cell lysates were prepared, proteins were resolved by gel electrophoresis, and EpCAM and matriptase were detected via immunoblotting with anti-HA and anti-Flag mAb, respectively. (B and C) Caco-2 cells were transfected with control siRNA or matriptase siRNAs using electroporation. Cell lysates that had been normalized for total protein concentrations were resolved using SDS-PAGE and matriptase, and EpCAM and claudin-7 expression was assessed using Western blotting. The data shown are representative of 1 of $3(\mathbf{A})$ or 4 (B and $\mathbf{C}$ ) experiments.

To study effects of HAI-2 and HAI-2 mutant proteins in cells expressing "physiologic" levels of matriptase and EpCAM, we prepared stably transfected Caco-2 cells expressing control HAI-2 or the patient-associated point mutant HAI-2 Y163C and then inhibited expression of HAI-2 that was encoded by the endogenous SPINT2 locus with siRNA complementary to untranslated sequences contained within mRNA derived from the endogenous locus but not in mRNA transcribed from the expression plasmid cDNA. The feasibility of this approach is documented in Figure $7 \mathrm{~B}$ and Supplemental Figure 4A, in which stably transfected G418-resistant pools or clones of Caco-2 cells were treated with SPINT2 siRNA and effects on HAI-2, EpCAM, and claudin-7 were assessed. Treatment of vectortransfected Caco-2 cells with SPINT2 siRNA inhibited endogenous HAI-2 expression, enhanced EpCAM cleavage, and destabilized claudin-7 in pooled cells (Figure 7B and Supplemental Figure 4A). In contrast, HAI-2 expression that was encoded by expression plasmids in pooled cells (Figure 7B) and each of several clones (Supplemental Figure 4A) was not inhibited by SPINT2 siRNA, EpCAM cleavage was not enhanced, and claudin-7 expression was maintained in Caco- 2 cells transfected with these constructs.

We subsequently compared the ability of control and CTE patient-associated mutant HAI-2 proteins to modulate matriptase-mediated EpCAM cleavage in analogous experiments. Clones of Caco-2 cells that had been stably transfected with control SPINT2 (WT1 and WT2) or SPINT2 Y163C (Y163C1 and Y163C2) were treated with SPINT2 siRNA, and effects on HAI2, EpCAM, claudin-7, and occludin were assessed via immunoblotting. Note that the levels of HAI-2 in the stable transfectants exceeded those present in vector-transfected Caco- 2 cells and that levels of HAI-2 mutant proteins in transfectants exceeded those in cells transfected with control SPINT2 (Figure 7C and Supplemental Figure 4B). Consistent with Figure 7B and Supplemental Figure $4 \mathrm{~A}$, knockdown of endogenous HAI-2 in Caco-2 cells transfected with control SPINT2 did not result in enhanced EpCAM cleavage or decreased claudin-7 expression. Despite being expressed at levels equal to or exceeding those of corresponding control proteins, in the absence of endogenous HAI-2, HAI-2 Y163C failed to inhibit matriptase-mediated proteolysis of EpCAM and did not stabilize claudin-7. The relative inability of CTE-associated HAI-2 proteins (Y163C or G168S) to stabilize claudin-7 in IECs was also observed in experiments involving pools of stably transfected Caco-2 cells where levels of expression of claudin-7 and control or mutant HAI2 proteins were assessed simultaneously via confocal immunofluorescence microscopy analysis of Transwell cell monolayers (Figure 7D and Supplemental Figure 4C).

\section{Discussion}

Congenital tufting enteropathy (CTE) features severe intestinal epithelial dysplasia with compromise of the intestinal epithelial barrier. Normal intestinal epithelial homeostasis requires carefully choreographed continual remodeling of apical junctional complexes that are composed of tight junctions, gap junctions, adherens junctions, and desmosomes. Junctional complexes mediate adhesion of IECs to each other and maintain barrier function $(32,33)$. Previous studies have identified the cell surface protease matriptase as an important regulator of tight junction composition and function, but detailed molecular mechanisms that link matriptase and tight junctions have not been delineated (21, 22). EpCAM also modulates tight junctions, albeit in a reciprocal fashion (10, 12). Whereas inhibition of expression of matriptase in Caco- 2 cell monolayers increased permeability and decreased transepithelial electrical resistance (TEER) (21), inhibition of EpCAM expression by Caco- 2 cells enhanced TEER in conjunction with decreased claudin-7 expression (12). The observation that SPINT2, EPCAM, and $C l d n 7$ mutations cause CTE in humans or equivalent phenotypes in mice, and the known inverse relationship between matriptase activity and expression of HAI-2, led us to explore the possible participation of these genes and corresponding proteins in a pathway that could regulate intestinal tissue and cell homeostasis.

Herein we demonstrate that EpCAM is one of a few physiologically relevant matriptase substrates that have been identified to date. Active matriptase, but not prostasin, introduced a single cleavage into EpCAM after Arg80 in solution and in cells. Labeling studies indicated that both full-length EpCAM and cleaved EpCAM were exposed on Caco-2 cell surfaces and that the $36-\mathrm{kDa}$ EpCAM fragment was preferentially internalized and targeted for 
A
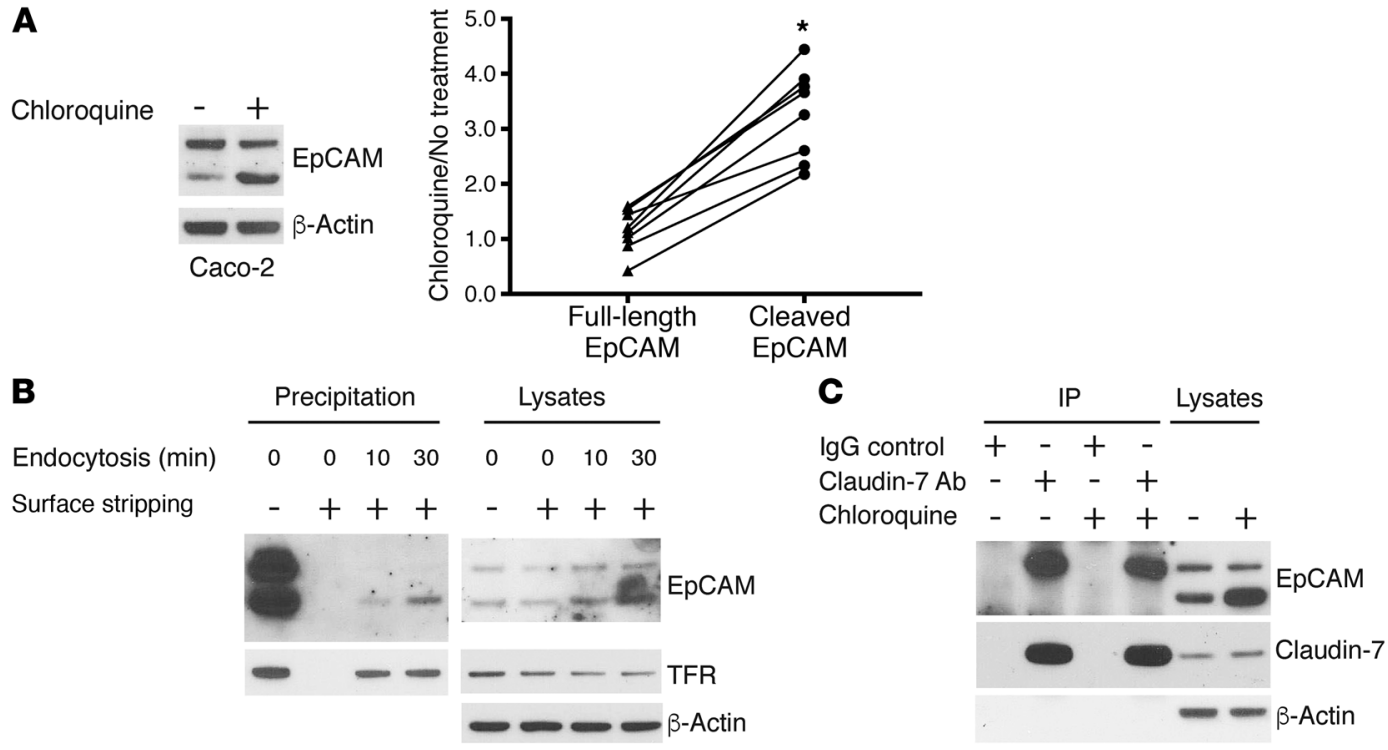

Figure 5. Matriptase cleavage leads to dissociation of EPCAM and claudin-7 and targets EpCAM for internalization and lysosomal degradation. (A) Caco-2 cells were treated with or without $100 \mu \mathrm{M}$ chloroquine for 20 hours, and RIPA lysate proteins were resolved using SDS-PAGE and immunoblotted with anti-EpCAM. Band intensities corresponding to full-length EpCAM and matriptase-cleaved EpCAM were quantified and normalized to that corresponding to $\beta$-actin intensity in each condition. Paired ratios of band intensities after chloroquine treatment relative to nontreatment controls are depicted $(n=8)$. The 2-tailed $P$ value ( $\left.{ }^{*} P<0.0001\right)$ for the comparison of abundances of full-length EpCAM and cleaved EpCAM in chloroquine-treated and untreated cells was determined using a paired $t$ test. (B) Caco-2 cells were labeled with sulfo-NHS-SS-biotin for 30 minutes at $4^{\circ} \mathrm{C}$, followed by incubation at $37^{\circ} \mathrm{C}$ for the indicated times to allow cell surface proteins to be internalized. Cell surface biotin was stripped via treatment with MESNA, cell lysates were prepared, and biotin-labeled proteins were recovered as described in Methods. Proteins were resolved using SDS-PACE and immunoblotted with anti-EpCAM or anti-transferrin receptor (TFR). Representative data from 1 of 3 experiments are shown. (C) Caco-2 cells treated with or without $100 \mu \mathrm{M}$ chloroquine for 20 hours were Iysed, protein concentrations were normalized, and immunoprecipitations were carried out with anti-claudin-7 Ab or IgG. Immunoprecipitates and lysate proteins were resolved with SDS-PAGE and immunoblotted with anti-EpCAM and anti-claudin-7. Representative data from 1 of 3 experiments are shown.

degradation (with claudin-7) in lysosomes. The existence of significant amounts of 36-kDa EpCAM on IEC surfaces suggests that cleavage of EpCAM does not lead to rapid internalization, or perhaps that cleaved EpCAM can be recycled from endosomes back to cell surfaces. Interestingly, coimmunoprecipitation revealed that claudin-7 exclusively associated with uncleaved EpCAM. We also showed that, in HEK293 and Caco-2 cells, control HAI-2 efficiently inhibited matriptase-dependent EpCAM proteolysis while a CTE-associated HAI-2 point mutant protein did not. In aggregate, these observations indicate that HAI-2, matriptase, EpCAM, and claudin-7 are directly linked in a functionally important pathway. Normal intestinal homeostasis requires that matriptase activity be restrained by HAI-2, which in turn prevents excessive cleavage of EpCAM and inappropriate degradation of EpCAM and claudins (including claudin-7). The existence of this pathway also explains why mutations in any of SPINT2, EPCAM (Epcam), or Cld7 can cause CTE (Figure 8).

The EpCAM processing pathway that we have characterized appears to correspond to that predicted by others almost 30 years ago (26) and to be distinct from the regulated intramembrane proteolysis of EpCAM associated with Notch-like signaling that was reported by Maetzel et al. (7). The latter investigators reported that the coordinate action of metalloproteases, $\beta$-secretases, and $\gamma$-secretases resulted in shedding of extracellular fragments of EpCAM (EpEX) and release of soluble transcription-modifying intracellular domains (EpICD) (7). It is also not obvious that our observations relate to the previously described ability of EpCAM to augment Wnt signaling by preventing degradation of Wnt coreceptors such as LPR5/6 (9). Perhaps these activities of EpCAM are germane to the biology of carcinomas and/or stem cells, while the HAI-2/matriptase/EpCAM/claudin-7 pathway that we have elucidated is relevant primarily in differentiated epithelial cells in the context of intracellular adhesion.

An intersection of the HAI-2/matriptase/EpCAM/claudin-7 pathway with the atypical PKC-modifying activity of EpCAM is possible. The intracellular domain of EPCAM is homologous to autoinhibitory pseudosubstrate domains of PKCs, and, in cells where atypical PKC and EpCAM are coexpressed, EpCAM can bind directly to, and inhibit the activity of, atypical PKC (8). If this mechanism is operative in intestinal epithelia, loss of EpCAM expression secondary to a SPINT2 or an EPCAM mutation would be predicted to lead to increased atypical PKC activity and, potentially, downstream effects on intercellular adhesion and/or cell migration. The observation that decreased expression of matriptase in Caco-2 cells, and hence reduced EpCAM cleavage and degradation, is associated with decreased atypical PKC activity (8) is consistent with this concept. In addition, Tsukita and colleagues have reported that conditional inactivation of $C l d n 7$ in murine intestinal epithelia leads to downregulation of EpCAM expression via a posttranscriptional mechanism (14). Thus, loss of EpCAM in this setting could also result in tissue-specific activation of atypical PKC. Although it is clear that the HAI-2/matriptase/EpCAM/claudin-7 pathway is operative and relevant in differentiated IECs, we have not addressed its importance in stem cells or tumor cells. In future studies, it will be interesting to 
A

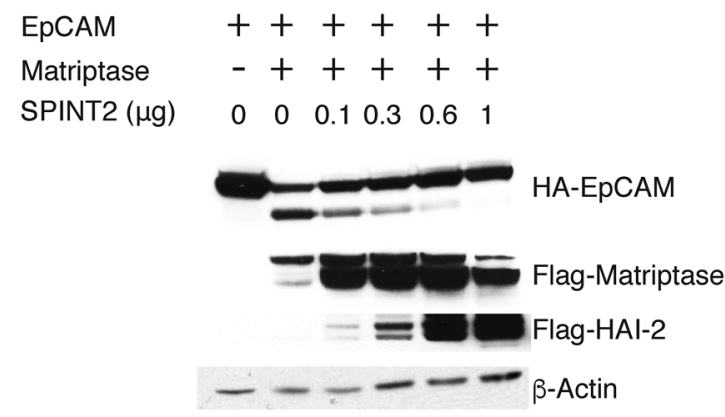

B

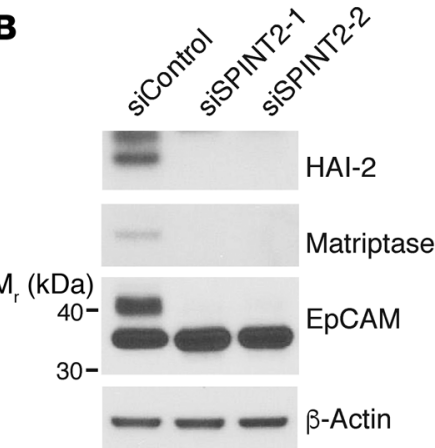

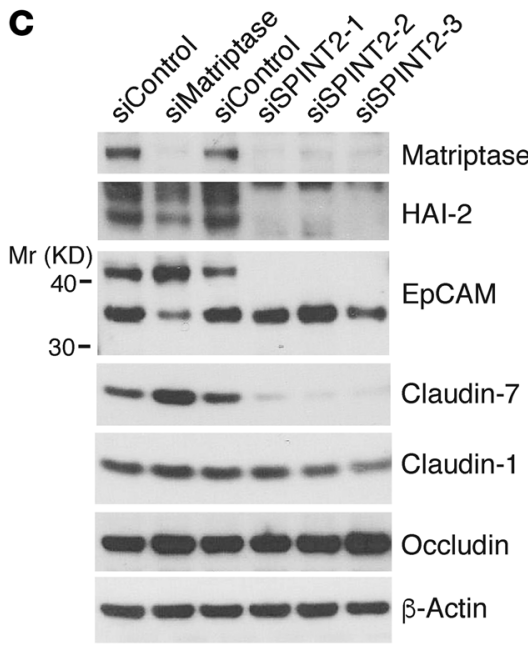
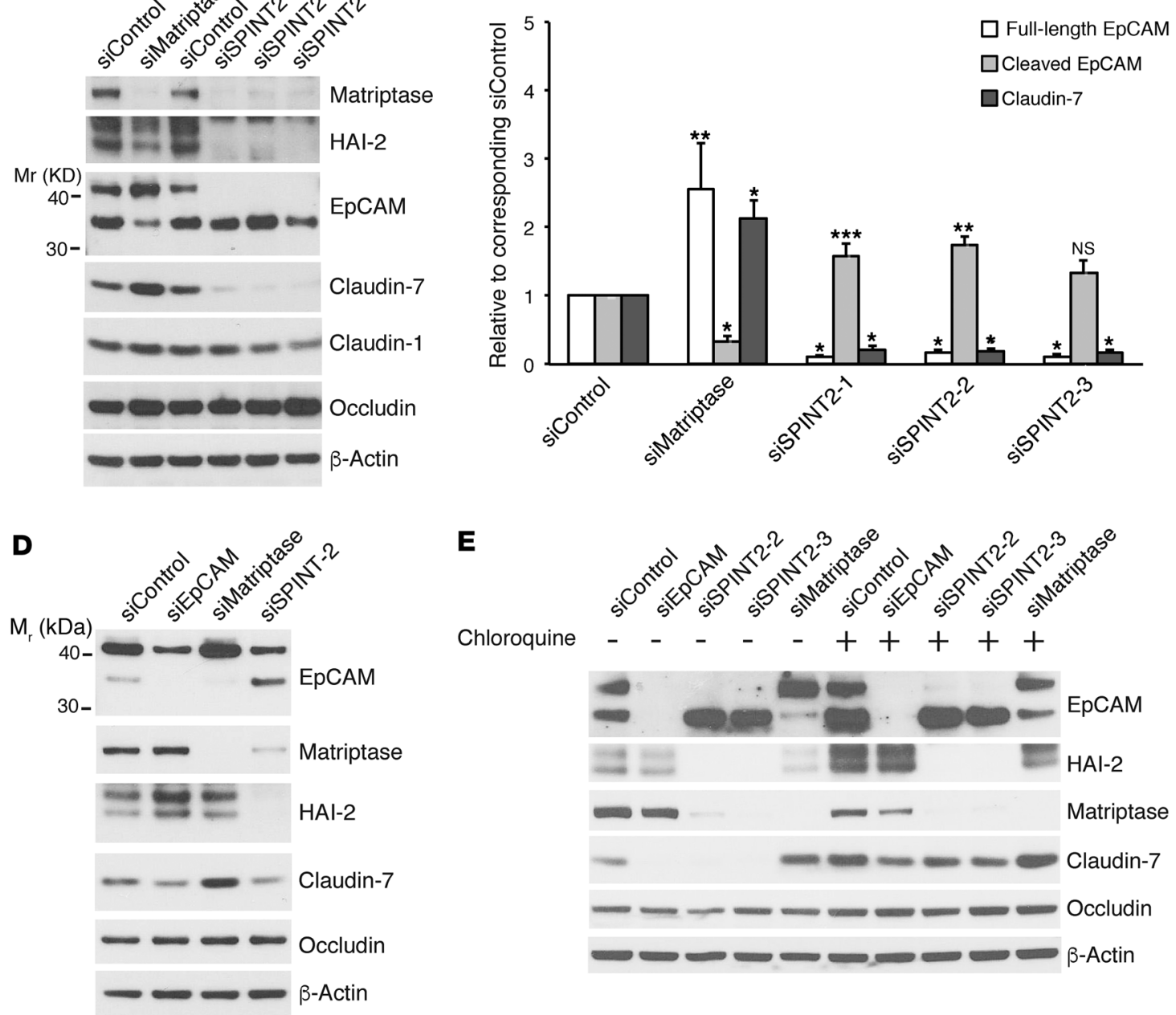

Figure 6. HAI-2 prevents EPCAM cleavage and subsequent claudin degradation in lysosomes by inhibiting matriptase. (A) HEK293 cells were transiently transfected with fixed amounts of pcDNA3 encoding EpCAM and matriptase and varied amounts of SPINT2 expression plasmids as indicated. Proteins of interest were quantified in cell lysates via immunoblotting using relevant Ab. (B-D) Caco-2 cells (B and C) and T84 cells (D) were transfected with control siRNA, EpCAM siRNA, matriptase siRNA, or SPINT2 siRNAs via electroporation. Transfected cells were replated the next day and cultured for 2 more days. Cell lysate proteins were resolved using SDS-PAGE and immunoblotted with anti-matriptase, anti-HAI-2, anti-EpCAM, anti-claudin-7, anti-claudin-1, or anti-occludin as indicated. Claudin-7 and EpCAM band intensities in C were quantified and normalized to $\beta$-actin signals. Data are depicted as ratios (mean \pm SEM) relative to corresponding siControls $(n=5)$. A repeated-measures 2-ANOVA Dunnett's method was used to calculate 2 -tailed $P$ values corrected for multiple comparisons to assess mean differences between groups $\left({ }^{*} P<0.0001,{ }^{*} P<0.005\right.$, $\left.{ }^{* * *} P<0.05\right)$. (E) Caco-2 cells were transfected with control siRNA, EpCAM siRNA, matriptase siRNA, or SPINT2 siRNA, and treated with or without $100 \mu \mathrm{M}$ chloroquine for 20 hours. RIPA lysates were subjected to SDS-PAGE and analyzed for EpCAM, HAI-2, claudin-7, and occludin via Western blotting. $\beta$-Actin was used as a loading control. Representative data from 1 of $3(\mathbf{A}, \mathbf{D}$, and $\mathbf{E})$ or 1 of 5 (B and $\mathbf{C})$ experiments are shown. 


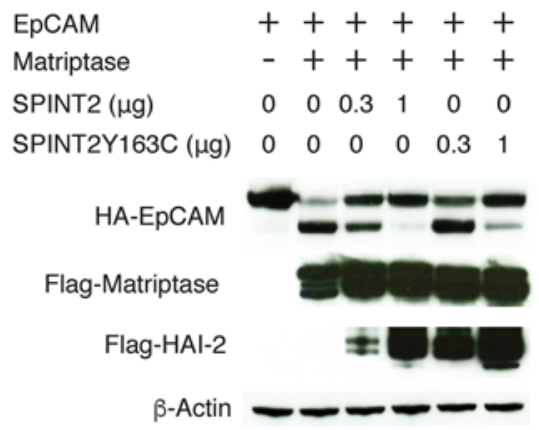

B

siControl

siSPINT2-2

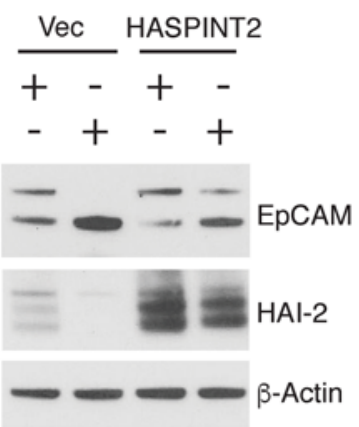

C

siControl $\frac{\text { Vec }}{+-} \frac{\text { WT1 }}{+-} \frac{\text { WT2 }}{+-} \frac{\text { Y163C1 }}{+-} \frac{\text { Y163C2 }}{+-}$

sisPINT2-2
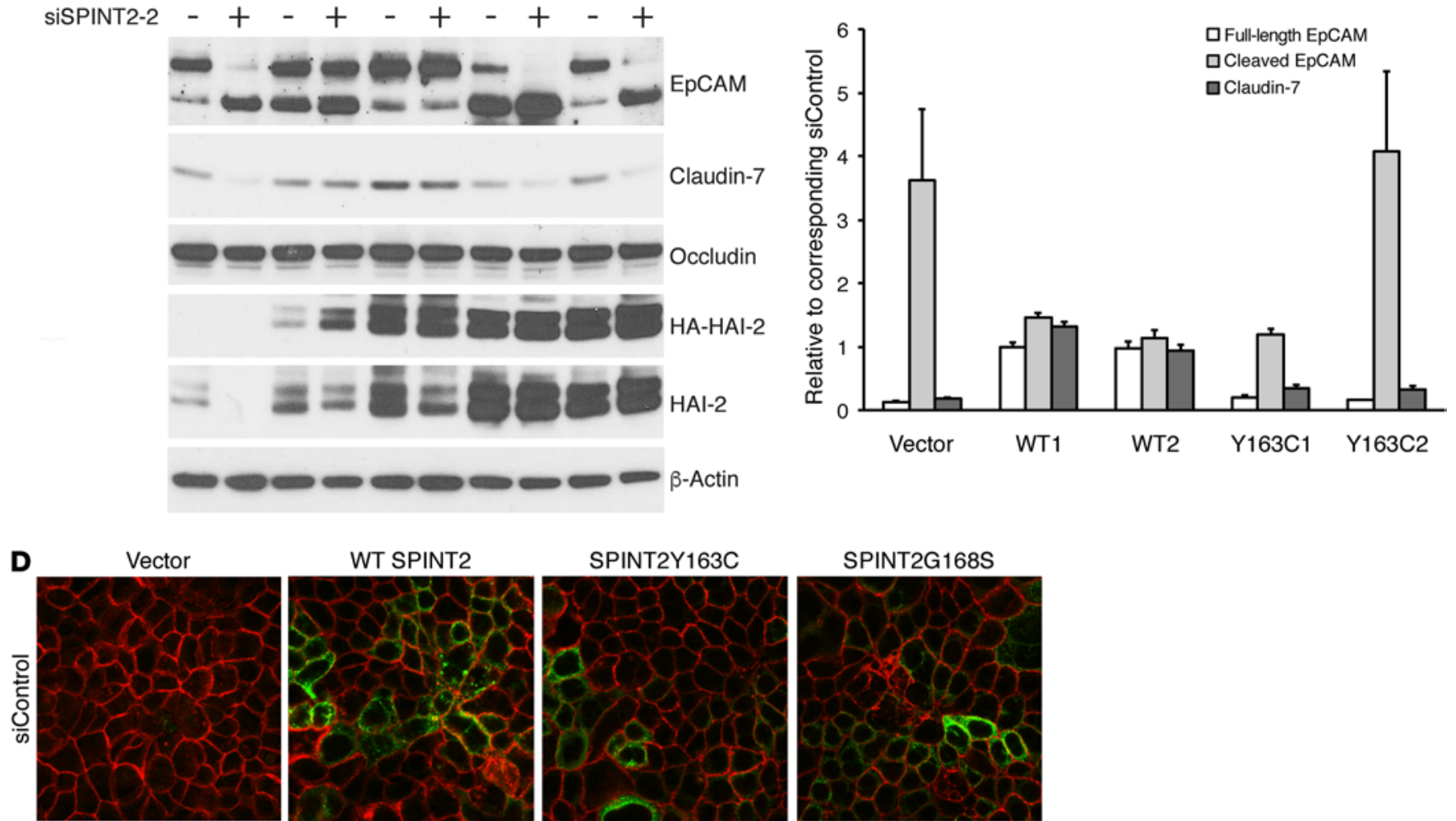

SPINT2G168S
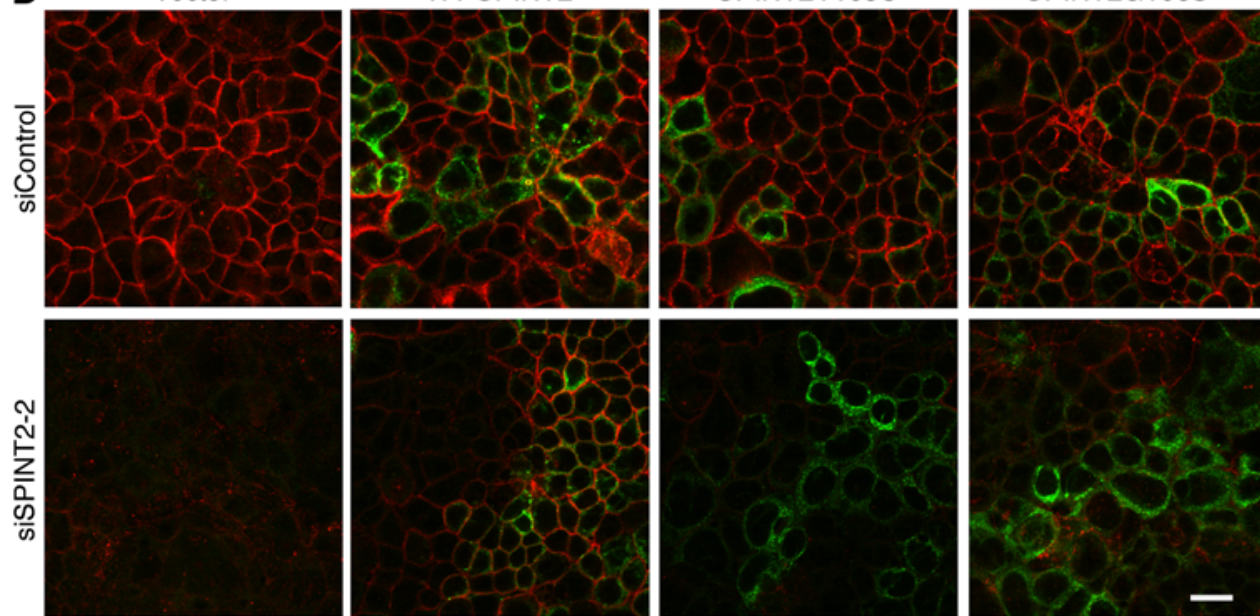

Green, HA-HAI-2; red, claudin-7
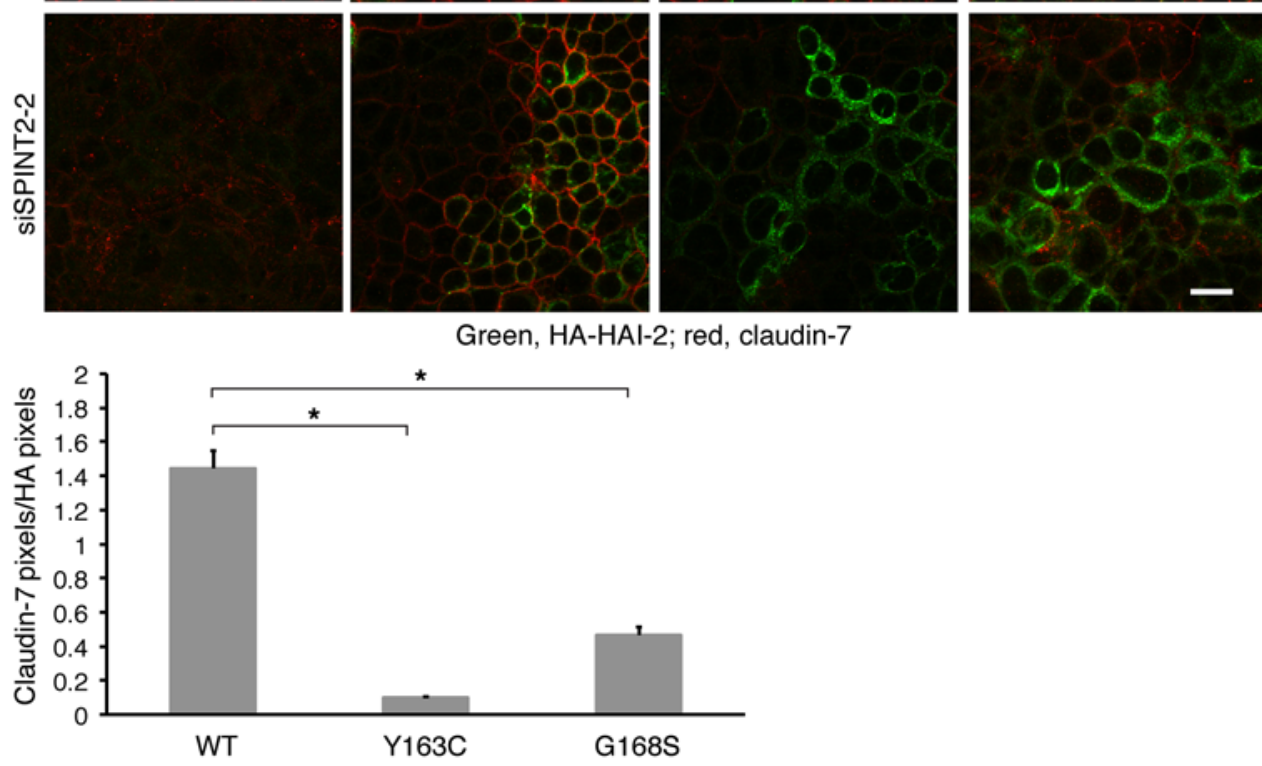
Figure 7. CTE-associated HAI-2 mutant proteins are less potent inhibitors of matriptase-mediated EpCAM cleavage and claudin degradation than wildtype HAI-2. (A) HEK293 cells were transiently transfected with fixed amounts of pcDNA3 encoding EpCAM and matriptase and varied amounts of pcDNA3 encoding wild-type or Y163C mutant SPINT2. After 48 hours, EpCAM, matriptase, and HAI-2 were detected by Western blotting. (B-D) Caco-2 cells were transfected with pcDNA3 or pcDNA3 encoding HA-tagged wild-type SPINT2, SPINT2 Y163C, or SPINT2 G168S and selected with G418 for 2 weeks. Surviving cells were pooled (B and D) or cloned (C), and HAl-2 expression was assessed via immunoblotting with anti-HA and anti-HAl-2. Pooled cells (B) or cloned cells (C) transfected with plasmids encoding wild-type HAI-2 or HAl-2 mutant proteins were transfected with control siRNA or SPINT2 siRNA (targeting the 5 ' untranslated region of SPINT2 mRNA). Cell lysates were harvested 72 hours later and immunoblotted with anti-EpCAM, anti-claudin-7, anti-occludin, anti-HA, anti-HAl-2, or anti- $\beta$-actin. Representative data from 1 of 3 experiments is shown in $\mathbf{A}$ and $\mathbf{B}$. Band intensities corresponding to claudin-7 and EpCAM in (C) were quantified and normalized to $\beta$-actin. Data are depicted as mean \pm SEM of ratios of signals corresponding to full-length EpCAM, cleaved EpCAM, and claudin-7 relative to those in control siRNA transfections $(n=4)$. $P$ values of comparisons between wild-type HASPINT2 and HASPINT2 Y163C transfected cells were determined as described in Supplemental Figure 4B. (D) Pooled Caco-2 cells transfected with vector, SPINT2, SPINT2 Y163C, or SPINT2 G168S plasmids were transiently transfected with control siRNA or SPINT2 siRNA. siRNA transfected cells were replated into Transwells the next day and cultured for 5 additional days to establish monolayers. Methanol-fixed monolayers were stained with anti-HA (green) or anti-claudin-7 (red) and analyzed using confocal microscopy. Scale bar: $20 \mu \mathrm{m}$. Representative images from 1 of 3 experiments are presented. For the experiment depicted, 10 images of SPINT2 siRNA transfected wild-type HASPINT2, HASPINT2 Y163C, or HASPINT2 G168S expressing cells were subjected to quantitative analysis. Values depicted represent mean \pm SEM of claudin-7 pixels/HA-HAl-2 pixels ( $n=10$, each image including at least 50 cells, for each experimental condition). Two-tailed $P$ values for the comparisons between effects of individual mutant (Y163C or G168S) and wild-type constructs were calculated via 1-way ANOVA using Dunnett's method $\left({ }^{*} P<0.0001\right)$.

determine whether manipulation of this pathway in small intestinal epithelial organoids (34) and/or in stem cell-mimicking spheroids (35) results in a dramatic phenotype.

We have concluded that EpCAM is a matriptase substrate on the basis of our determination that (a) EpCAM and matriptase colocalize in IECs in vivo and in vitro; (b) EpCAM and matriptase coimmunoprecipitate from Caco-2 cells that have been treated with a protein cross-linking reagent and from 293 cells that overexpress EpCAM and matriptase; (c) EpCAM is cleaved in 293 cells when it is cointroduced with matriptase; and (d) in solution, recombinant EpCAM is selectively and appropriately cleaved by recombinant matriptase and not recombinant prostasin (another cell surface serine protease). The latter observation is somewhat surprising and perhaps particularly meaningful because proteases can be promiscuous in cell-free in vitro assays.

We have not specifically addressed the mechanism of matriptase activation in IECs in the absence of HAI-2, but this has been studied extensively by Bugge and colleagues (and others as well; refs. 16, 18, 20). In intestine, prostasin and matriptase form a reciprocal zymogen complex that results in formation of active matriptase and active prostasin (36). At baseline, the activity of the prostasin/matriptase complex is coordinately regulated by HAI- 1 and HAI-2 (37). HAI-2 appears to directly inhibit active prostasin, thereby preventing matriptase activation. In the absence of HAI-2, basal prostasin activity is sufficient to efficiently activate matriptase (16). Consequences of matriptase activation in IECs in the absence of HAI-2 include cleavage of EpCAM and lysosomal degradation of selected claudins, including claudin-7.

During the course of these studies, we observed that a single cleavage in the N-terminus of EpCAM prevents tight association of EpCAM with claudin-7. Although the direct interaction of EpCAM with claudin-7 has not been formally demonstrated, the relative paucity of other proteins in preparative anti-EpCAM immunoprecipitates from Caco- 2 cells suggests that this is the case $(12,38)$. Previous studies have demonstrated that EpCAMclaudin-7 interactions are strongly influenced by amino acid substitutions at 2 positions within the EpCAM transmembrane domain (39). We have confirmed these results (12) but currently have no insights into mechanisms that might link $\mathrm{N}$-terminal cleavage of EpCAM to conformational changes in the EpCAM transmem- brane domain and dissociation of claudin-7 from EpCAM. We also do not yet understand why cleavage of EpCAM after Arg80 targets EpCAM for internalization and lysosomal degradation along with EpCAM-associated claudin-7. We note with great interest that EpCAM is destabilized in the intestinal epithelium of $\mathrm{Cldn} 7$ conditional knockout mice (14). In normal IECs, EpCAM and claudin-7 apparently stabilize each other in a reciprocal relationship that requires additional characterization.

We propose that the HAI-2/matriptase/EpCAM/claudin-7 pathway that we have elucidated is of fundamental importance for epithelial homeostasis. Although several of the proteins in this pathway are widely expressed, it is interesting to note that the predominant phenotypes of humans and mice with EPCAM and SPINT2 mutations are expressed in the gut. One possible explanation for this observation is that proteolysis involving prostasin and matriptase may be regulated via tissue-specific mechanisms. For example, in skin matriptase appears to be an upstream activator of prostasin (18), whereas prostasin activates matriptase in the gastrointestinal tract (36). Another possibility is that intestinal epithelium is one of only a few tissues where the EpCAM homolog TROP2 is not coexpressed with EpCAM (40). If EpCAM and TROP2 are functionally redundant, intestinal epithelium would be expected to be particularly vulnerable to loss of EpCAM. Future studies will address the functional relationship of EPCAM and TROP2, and the participation of matriptase in the regulation of TROP2 function. We will also assess the degree to which the HAI-2/matriptase/EpCAM/ claudin-7 pathway is dysregulated in other pathologic settings that feature abnormal intestinal epithelial barrier function (such as inflammatory bowel disease) and in the setting of malignant transformation as well. Should overactive matriptase be documented in any of these settings, it is conceivable that it could be targeted via systemically administered or topically applied small-molecule or peptide-containing enzyme inhibitors. An analogous approach might benefit CTE patients with SPINT2 mutations.

\section{Methods}

Cells. Caco- 2 cells, T84 cells, and Caco- 2 cells that are stable expressers of EpCAM shRNA have been described previously (12). Caco-2 cells were grown in DMEM containing 10\% FBS, 15 mM HEPES, and nonessential amino acids ( $\mathrm{pH}$ 7.4). T84 cells were cultured in DMEM/ 


\section{A Functional Pathway Linking HAI-2 (SPINT2),} Matriptase, EpCAM, and Selected Claudins

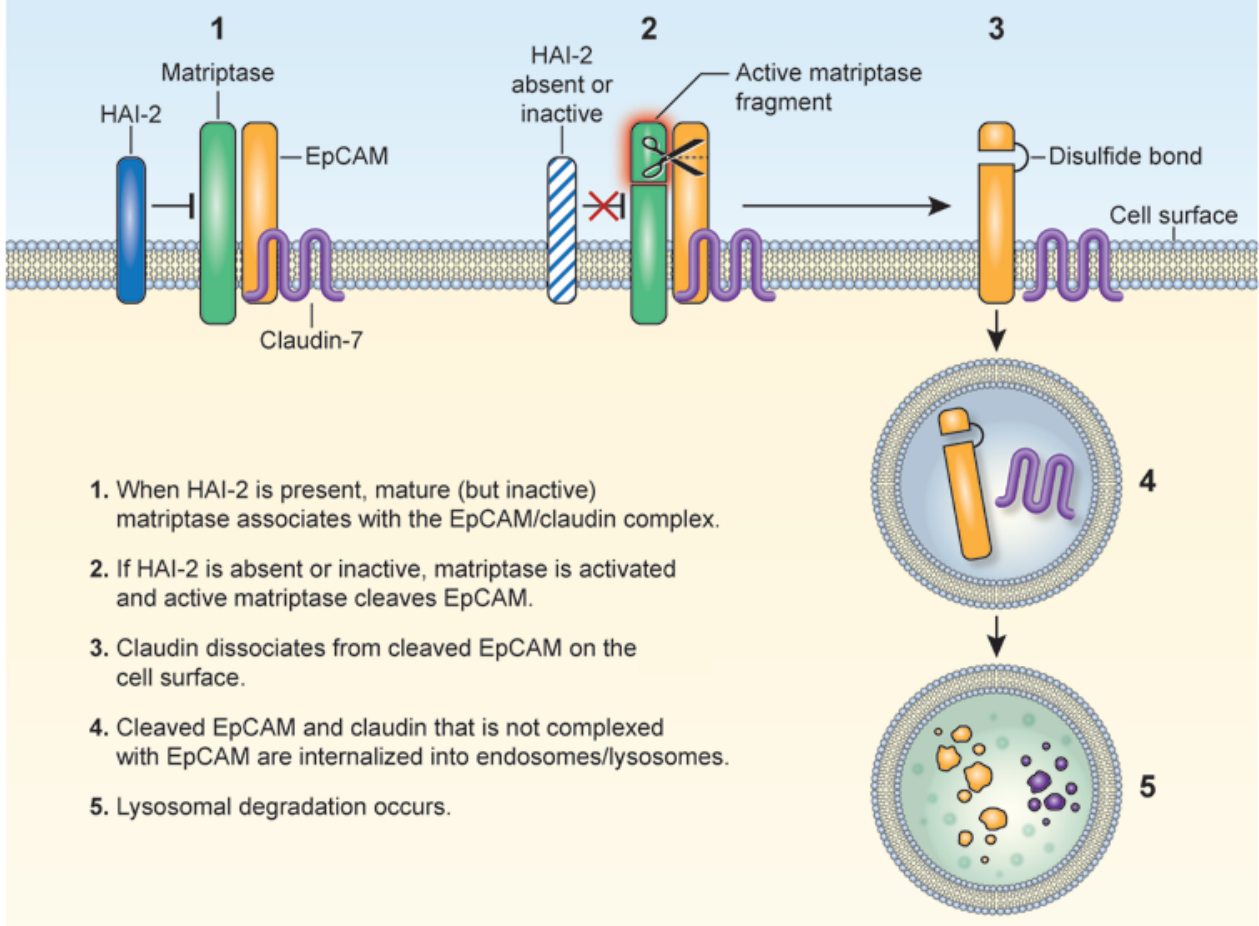

Figure 8. A functional pathway linking HAI-2/SPINT2, matriptase, EPCAM, and claudin-7. EpCAM complexes with claudin-7 and colocalizes with matriptase on the lateral surfaces of polarized IECs. CTE-associated mutations in SPINT2 inactivate the matriptase inhibitor HAI-2. Unrestrained active matriptase then cleaves EpCAM, leading to dissociation of EpCAM and claudin-7 followed by internalization and lysosomal degradation of both EpCAM and claudin-7. The existence of this pathway explains why mutations in SPINT2, EPCAM, and $C L D 7$ can cause very similar phenotypes.

F12 supplemented with 6\% FBS and 15 mM HEPES (pH 7.4). HEK293 cells were from the American Type Culture Collection. To establish polarized IEC monolayers, T84 cells or Caco- 2 cells $\left(6 \times 10^{5}\right.$ and $3 \times 10^{5}$ cells per well, respectively) were plated into Transwells with $12-\mathrm{mm}$ diameter polycarbonate filters and pore sizes of $0.4 \mu \mathrm{m}$ (Corning Costar). T84 and Caco-2 monolayers were cultured for 8-10 days or 21-25 days, respectively, with media changes every other day. Transepithelial electrical resistance (TEER) was monitored with an EVOM2 Epithelial Voltohmmeter (World Precision Instruments). Mouse small intestine organoids were generated using the method developed by Sato and Clevers (41).

Antibodies. Polyclonal rabbit anti-EpCAM Ab was generated by immunization of rabbits with recombinant protein composed of the extracellular domain of mouse EpCAM fused to the Fc region of human IgG as described previously (12). Mouse monoclonal anti-EpCAM Ab (clones EBA-1 and 323/A30) and goat anti-C-terminal EpCAM polyclonal Ab (sc-23788) were purchased from Santa Cruz Biotechnology. Anti-C-terminal EpCAM (PA5-19832), anti-claudin-1 (71-7800), anticlaudin-7 (34-9100), anti-occludin (71-1500), and anti-transferrin receptor rabbit polyclonal $\mathrm{Ab}$ (13-6800) and mouse anti-claudin-7 $\mathrm{mAb}$ (37-4800) were from Life Technologies. Rabbit anti-matriptase $\mathrm{Ab}$ (IM1014) was purchased from EMD Biosciences, and sheep anti-matriptase (AF3946) was from R\&D Systems. Polyclonal anti-
HAI-2 Ab (HPA011101), mouse anti- $\beta$-actin mAb (clone AC-15), rabbit anti-actin polyclonal $\mathrm{Ab}$ (SAB4301137), and mouse anti-Flag $\mathrm{mAb}$ (clone M2) were obtained from Sigma-Aldrich, and rat anti-HA mAb (clone 3F10) was from Roche.

Gene expression plasmids. pcDNA3-HAEpCAM has been described (12). Plasmids expressing human matriptase, Flag-tagged human matriptase, and Flag-tagged human HAI-2 were purchased from OriGene. HA-tagged SPINT2 cDNA was amplified by PCR using the reverse transcription products of Caco-2 total RNA as templates and cloned into pcDNA3 that had been digested with BamHI and XhoI. The resulting plasmid was verified by DNA sequencing. The SPINT2 coding sequence in the plasmid was identical to the published corresponding SPINT2 sequence (GenBank NM_021102). Mutations were introduced into the SPINT2 expression plasmid using a QuikChange Kit (Agilent Technologies) following the manufacturer's instructions.

Treatment of EpCAM with proteases. Catalytically active recombinant mouse matriptase and mouse prostasin were purchased from R\&D Systems. Recombinant mouse EpCAMhIgG protein was protein A-Sepharose (GE Healthcare) affinity-purified from medium that was conditioned by $293 \mathrm{~F}$ cells transfected with a plasmid encoding the mouse EpCAM extracellular domain fused in frame with the Fc portion of human IgG1 using Turbofect (Life Technologies) (12). Recombinant EpCAM was incubated with recombinant matriptase in $100 \mu \mathrm{l}$ "matriptase reaction buffer" (50 mM Tris, $\mathrm{pH}$ 8.5, $100 \mathrm{mM}$ $\mathrm{NaCl}$ ) or with recombinant matriptase or prostasin in $100 \mu \mathrm{l}$ "prostasin reaction buffer" (50 mM Tris, $0.05 \%$ Brij $35, \mathrm{pH} 9.5$ ) at $37^{\circ} \mathrm{C}$ for 1 hour as indicated. Enzymatic activities of recombinant matriptase and prostasin were verified using the fluorogenic peptide Boc-QAR-AMC and assay conditions recommended by the manufacturer.

Cell transfection and derivation of stable cell lines. Empty vectors or plasmids containing HA-tagged EpCAM, Flag-tagged matriptase, or Flag-tagged SPINT2 were introduced into 293 cells using Fugene 6 (Promega) following the manufacturer's instructions. pcDNA3 or pcDNA3 encoding SPINT2HA, SPINT2HA Y163C, or SPINT2HA G168S was transfected into Caco-2 cells using Lipofectamine 2000 (Life Technologies). Plasmid-expressing cells were selected with 700 $\mu \mathrm{g} / \mathrm{ml} \mathrm{G} 418$ for 14 days, and drug-resistant cells were pooled and analyzed for HAI-2 expression using immunoblotting. Selected individual stable clones were isolated and expanded.

Inhibition of protein expression with siRNAs. Double-stranded RNA oligonucleotides containing matriptase siRNAs (siMatriptase-1, 
HSS110268; siMatriptase-2, HSS186125) were purchased from Life Technologies. SPINT2 siRNAs (siSPINT2-1, Hs02_00354589; siSPINT2-2, Hs01_00168900; siSPINT2-3, Hs01_00168907) came from Sigma-Aldrich. EpCAM siRNA (sense oligo sequence, 5'-GUUUGCGGACUGCACUUCAdTdT-3') was synthesized by Qiagen. Negative control siRNAs were obtained from Qiagen and Sigma-Aldrich. Caco2 or T84 cells were transfected with negative control siRNA, matriptase siRNA, SPINT2 siRNA, or EpCAM siRNA duplexes via electroporation (Gene Pulser, Bio-Rad Laboratories) at $320 \mathrm{~V}$ (for T84) or 290 V (for Caco-2) for 20 seconds. Transfected cells were lysed 3-6 days after transfection, and protein levels and/or protein sizes were assessed by Western blotting.

Immunoblotting, immunoprecipitation, and coimmunoprecipitation. Cells were lysed with Triton X-100 lysis buffer or RIPA lysis buffer as indicated, and protein contents were normalized using a Bradford protein assay (Bio-Rad Laboratories) or BCA assay (Life Technologies) (12). For routine preparative immunoprecipitation, RIPA cell lysates were incubated with control IgG or Ab followed by precipitation with protein A- or protein G-Sepharose 4B Fast Flow beads (GE Healthcare). Protein associations were assessed via coimmunoprecipitation from TX-100 lysates as previously described (42). Proteins in immunoprecipitates or cell lysates were resolved by SDS-PAGE (Nupage BisTris gels, Life Technologies) under reducing conditions and visualized by Coomassie blue staining or Western blotting. For immunoblotting, proteins separated by SDS-PAGE were transferred onto nitrocellulose membranes that were subsequently incubated with the indicated $\mathrm{Ab}$. Proteins of interest were visualized using HRP-conjugated secondary $\mathrm{Ab}$ (Jackson ImmunoResearch) and enhanced chemiluminescence (Life Technologies). Intensities of protein bands were quantified using densitometry and ImageJ software (NIH).

$\mathrm{N}$-terminal protein sequencing. Anti-EpCAM immunoprecipitates of Caco-2 cell lysates or matriptase-treated recombinant mouse EpCAM-Ig were resolved using SDS-PAGE and transferred onto PVDF membranes, followed by staining with Colloidal Coomassie blue (Life Technologies). Bands were excised, and the N-terminal sequences of eluted proteins were determined using an Applied Biosystems 494 cLC Protein sequencer in a National Cancer Institute core facility. Phenylthiohydantoin derivatives of amino acids were analyzed on-line using an Applied Biosystems 785A/140C/610A chromatograph.

Surface protein endocytosis assay. Endocytosis and surface expression of EpCAM were assessed using a method described previously $(43,44)$ after modification. Caco-2 cells in $60-\mathrm{cm}$ dishes were placed on ice and washed twice with PBS containing $0.9 \mathrm{mM} \mathrm{CaCl}_{2}$ and 0.33 $\mathrm{mM} \mathrm{MgCl}{ }_{2}$ (PBS-CM). Cells were then incubated with cleavable membrane-impermeable sulfo-NHS-SS-biotin (Life Technologies) at a concentration of $0.5 \mathrm{mg} / \mathrm{ml}$ for 30 minutes on ice. After washing, residual reactive biotin was quenched using $50 \mathrm{mM} \mathrm{NH}_{4} \mathrm{Cl}$ in PBS-CM. Warm complete growth medium was added, and cells were warmed to $37^{\circ} \mathrm{C}$ for the times indicated to allow endocytosis to occur. Cells were then placed on ice, and surface (non-endocytosed) biotin was stripped by reduction with $100 \mathrm{mM}$ 2-mercaptoethanesulfonate (MESNA) in Tris-buffered saline supplemented with calcium and magnesium via three 10-minute treatments. Free sulfhydryl groups were then quenched by incubation of cells with $5 \mathrm{mg} / \mathrm{ml}$ iodoacetamide via three 5 -minute treatments. Cells were washed twice more with PBS-CM and then lysed in $1.25 \%$ Triton X-100, 0.25\% SDS, 50 mM Tris-HCl, pH 8.0, 150 mM NaCl, 5 $\mathrm{mM}$ EDTA, $5 \mathrm{mg} / \mathrm{ml}$ iodoacetamide containing Roche protease inhib- itor cocktail. Biotinylated proteins were collected by incubation with NeutrAvidin beads (Life Technologies), and beads were washed 5 times with $0.5 \%$ Triton X-100, 0.1\% SDS, 50 mM Tris-HCl, pH 8.0, $150 \mathrm{mM}$ $\mathrm{NaCl}, 1 \mathrm{mM}$ EDTA. Affinity-purified biotinylated proteins were resolved using SDS-PAGE and analyzed using Western blotting.

Immunofluorescence microscopy and image analysis. IECs grown on Transwell filters were fixed with cold acetone/ethanol (3:1) or cold methanol for 10 minutes. After blocking with $1 \%$ BSA, cells were stained with the indicated primary Ab or control IgG, followed by Alexa Fluor 488- or Alexa Fluor 568-conjugated secondary Ab (Life Technologies). Frozen sections of human small intestine were fixed with cold methanol. After blocking with 3\% dry milk (Bio-Rad Laboratories) including $5 \%$ normal donkey serum or $5 \%$ normal goat serum (as appropriate) for 1 hour at room temperature, sections were stained with $\mathrm{Ab}$ of interest followed by Alexa Fluor 488- or Alexa Fluor 568-conjugated secondary Ab. Stained cells or tissue sections were mounted in ProLong Gold antifade reagent containing DAPI (Life Technologies) before visualization with an LSM 780 confocal laser scanning microscope (Zeiss) and analysis with LSM Image Browser 4.0. Image intensity quantification was performed using Zeiss ZEN 2012 software. In these experiments, a blinded observer identified cells that expressed HA-HAI-2 and quantified levels of expression (pixels) corresponding to HA-HAI-2 and endogenous claudin-7 in HA-HAI-2-expressing cells. Calculation of ratios of claudin-7- and HA-HAI-2-specific immunofluorescence allowed objective comparisons of the abilities of wild-type and CTE-associated mutant HA-HAI-2 to stabilize claudin-7.

Statistics. As appropriate, raw data were transformed before analysis. The function that produced the best fit for the ANOVA model that was used in each circumstance was selected using Box-Cox power analysis. Dunnett's test was used to calculate 2-tailed $P$ values to analyze differences between groups as indicated. $P$ values of 0.05 or less were considered significant. Averaged results of multiple experiments are presented as the arithmetic mean \pm SEM.

Study approval. Mice were housed in a pathogen-free American Association for Laboratory Animal Care-accredited facility on the NIH campus in Bethesda, Maryland, USA. Animal studies were conducted in accordance with guidelines established by Research Animal Resource Center, NIH, under the guise of protocols approved by the National Cancer Institute Animal Care and Use Committee. The use of anonymous discarded surgical specimens of human intestine was approved by the Office of Human Subjects Research Protection, NIH.

\section{Author contributions}

CJW and MCU designed the project and the experiments. CJW performed most of the experiments. CJW, XF, ML, and SM conducted experiments, acquired data, and participated in data analysis. CJW and MCU carried out most of the data analysis and wrote the manuscript with input from XF, ML, and SM.

\section{Acknowledgments}

We thank Susan Garfield for image quantification and Langston Lim (Laboratory of Experimental Carcinogenesis, National Cancer Institute) for help with confocal microscopy; Oleg Chertov (Protein Chemistry Laboratory, Advanced Technology Program, SAIC-Frederick Inc., National Cancer Institute) for protein Nterminal sequence data; the Laboratory of Pathology Tissue Pro- 
cessing and Procurement Facility for providing human small intestinal tissue; Seth Steinberg and David Venzon (Biostatistics and Data Management Section, National Cancer Institute) for conducting the statistical analyses; and Isaac Brownell (National Cancer Institute), Matthew Ciorba (Washington University), Andrew Kowalczyk (Emory University), Takeshi Ouchi (Keio University), and Keisuke Nagao (National Cancer Institute) for helpful discussions.
This research was supported by the Intramural Research Program of the NIH, Center for Cancer Research, National Cancer Institute.

Address correspondence to: Mark C. Udey, Dermatology Branch, Center for Cancer Research, NCI, NIH, Building 10, Room 12N240E, Bethesda, Maryland 20892-1908, USA. Phone: 301.496.2481; E-mail: udeym@mail.nih.gov.
1. Sivagnanam M, et al. Identification of EpCAM as the gene for congenital tufting enteropathy. Gastroenterology. 2008;135(2):429-437.

2. Salomon J, et al. Genetic characterization of congenital tufting enteropathy: epcam associated phenotype and involvement of SPINT2 in the syndromic form. Hum Genet. 2014;133(3):299-310.

3. Mueller JL, McGeough MD, Peña CA, Sivagnanam M. Functional consequences of EpCam mutation in mice and men. Am J Physiol Gastrointest Liver Physiol. 2014;306(4):G278-G288.

4. Baeuerle PA, Gires O. EpCAM (CD326) finding its role in cancer. Br JCancer. 2007;96(3):417-423.

5. Martowicz A, Seeber A, Untergasser G. The role of EpCAM in physiology and pathology of the epithelium. Histol Histopathol. 2016;31(4):349-355.

6. Litvinov SV, Velders MP, Bakker HA, Fleuren GJ, Warnaar SO. Ep-CAM: a human epithelial antigen is a homophilic cell-cell adhesion molecule. JCell Biol. 1994;125(2):437-446.

7. Maetzel $D$, et al. Nuclear signalling by tumourassociated antigen EpCAM. Nat Cell Biol. 2009;11(2):162-171.

8. Maghzal N, Kayali HA, Rohani N, Kajava AV, Fagotto F. EpCAM controls actomyosin contractility and cell adhesion by direct inhibition of PKC. Dev Cell. 2013;27(3):263-277.

9. Lu H, Ma J, Yang Y, Shi W, Luo L. EpCAM is an endoderm-specific Wnt derepressor that licenses hepatic development. Dev Cell. 2013;24(5):543-553.

10. Lei Z, et al. EpCAM contributes to formation of functional tight junction in the intestinal epithelium by recruiting claudin proteins. Dev Biol. 2012;371(2):136-145.

11. Guerra E, et al. mTrop1/Epcam knockout mice develop congenital tufting enteropathy through dysregulation of intestinal E-cadherin/ $\beta$-catenin. PLoS One. 2012;7(11):e49302.

12. Wu CJ, Mannan P, Lu M, Udey MC. Epithelial cell adhesion molecule (EpCAM) regulates claudin dynamics and tight junctions. J Biol Chem. 2013;288(17):12253-12268.

13. Ding L, et al. Inflammation and disruption of the mucosal architecture in claudin-7-deficient mice. Gastroenterology. 2012;142(2):305-315.

14. Tanaka H, Takechi M, Kiyonari H, Shioi G, Tamura A, Tsukita S. Intestinal deletion of Claudin-7 enhances paracellular organic solute flux and initiates colonic inflammation in mice. Gut. 2015;64(10):1529-1538.

15. Szabo R, Hobson JP, Christoph K, Kosa P, List K, Bugge TH. Regulation of cell surface protease matriptase by HAI2 is essential for placental development, neural tube closure and embryonic survival in mice. Development.
2009;136(15):2653-2663.

16. Friis S, Sales KU, Schafer JM, Vogel LK, Kataoka $\mathrm{H}$, Bugge TH. The protease inhibitor HAI-2, but not HAI-1, regulates matriptase activation and shedding through prostasin. J Biol Chem. 2014;289(32):22319-22332.

17. Lin CY, Anders J, Johnson M, Sang QA, Dickson RB. Molecular cloning of cDNA for matriptase, a matrix-degrading serine protease with trypsin-like activity. J Biol Chem. 1999;274(26):18231-18236.

18. Miller GS, List K. The matriptase-prostasin proteolytic cascade in epithelial development and pathology. Cell Tissue Res. 2013;351(2):245-253.

19. Parr C, Jiang WG. Hepatocyte growth factor activation inhibitors (HAI-1 and HAI-2) regulate HGF-induced invasion of human breast cancer cells. Int J Cancer. 2006;119(5):1176-1183.

20. Lin CY, et al. Zymogen activation, inhibition, and ectodomain shedding of matriptase. Front Biosci. 2008;13:621-635.

21. Buzza MS, et al. Membrane-anchored serine protease matriptase regulates epithelial barrier formation and permeability in the intestine. Proc Natl Acad Sci U S A. 2010;107(9):4200-4205.

22. List $\mathrm{K}$, et al. Epithelial integrity is maintained by a matriptase-dependent proteolytic pathway. Am J Pathol. 2009;175(4):1453-1463.

23. Netzel-Arnett $S$, et al. Matriptase protects against experimental colitis and promotes intestinal barrier recovery. Inflamm Bowel Dis. 2012;18(7):1303-1314.

24. Kosa P, Szabo R, Molinolo AA, Bugge TH. Suppression of Tumorigenicity-14, encoding matriptase, is a critical suppressor of colitis and colitis-associated colon carcinogenesis. Oncogene. 2012;31(32):3679-3695.

25. List K. Matriptase: a culprit in cancer? Future Oncol. 2009;5(1):97-104.

26. Thampoe IJ, Ng JS, Lloyd KO. Biochemical analysis of a human epithelial surface antigen: differential cell expression and processing. Arch Biochem Biophys. 1988;267(1):342-352.

27. Szala S, Kasai Y, Steplewski Z, Rodeck U, Koprowski H, Linnenbach AJ. Molecular cloning of cDNA for the human tumor-associated antigen CO-029 and identification of related transmembrane antigens. Proc Natl Acad Sci U S A. 1990;87(17):6833-6837.

28. Hooper JD, Clements JA, Quigley JP, Antalis TM. Type II transmembrane serine proteases. Insights into an emerging class of cell surface proteolytic enzymes. J Biol Chem. 2001;276(2):857-860.

29. List K, et al. Matriptase/MT-SP1 is required for postnatal survival, epidermal barrier function, hair follicle development, and thymic homeostasis. Oncogene. 2002;21(23):3765-3779.
30. d'Apolito M, et al. Genetic analysis of Italian patients with congenital tufting enteropathy. World J Pediatr. 2016;12(2):219-224.

31. Faller N, Gautschi I, Schild L. Functional analysis of a missense mutation in the serine protease inhibitor SPINT2 associated with congenital sodium diarrhea. PLoS One. 2014;9(4):e94267.

32. Laukoetter MG, Bruewer M, Nusrat A. Regulation of the intestinal epithelial barrier by the apical junctional complex. Curr Opin Gastroenterol. 2006;22(2):85-89.

33. Turner JR, Buschmann MM, Romero-Calvo I, Sailer A, Shen L. The role of molecular remodeling in differential regulation of tight junction permeability. Semin Cell Dev Biol. 2014;36:204-212.

34. Sato T, et al. Single Lgr5 stem cells build cryptvillus structures in vitro without a mesenchymal niche. Nature. 2009;459(7244):262-265.

35. Miyoshi H, Stappenbeck TS. In vitro expansion and genetic modification of gastrointestinal stem cells in spheroid culture. Nat Protoc. 2013;8(12):2471-2482.

36. Buzza MS, Martin EW, Driesbaugh KH, Désilets A, Leduc R, Antalis TM. Prostasin is required for matriptase activation in intestinal epithelial cells to regulate closure of the paracellular pathway. J Biol Chem. 2013;288(15):10328-10337.

37. Friis $S$, et al. Transport via the transcytotic pathway makes prostasin available as a substrate for matriptase. J Biol Chem. 2011;286(7):5793-5802.

38. Ladwein M, et al. The cell-cell adhesion molecule EpCAM interacts directly with the tight junction protein claudin-7. Exp Cell Res. 2005;309(2):345-357.

39. Nübel T, et al. Claudin-7 regulates EpCAMmediated functions in tumor progression. Mol Cancer Res. 2009;7(3):285-299.

40. Nakatsukasa M, et al. Tumor-associated calcium signal transducer 2 is required for the proper subcellular localization of claudin 1 and 7: implications in the pathogenesis of gelatinous drop-like corneal dystrophy. Am J Pathol. 2010;177(3):1344-1355.

41. Sato T, Clevers H. Primary mouse small intestinal epithelial cell cultures. Methods Mol Biol. 2013;945:319-328.

42. Wu CJ, Conze DB, Li T, Srinivasula SM, Ashwell JD. Sensing of Lys 63-linked polyubiquitination by NEMO is a key event in NF-kappaB activation [corrected]. Nat Cell Biol. 2006;8(4):398-406.

43. Nishimura N, Sasaki T. Regulation of epithelia cell adhesion and repulsion: role of endocytic recycling. JMed Invest. 2008;55(1-2):9-16.

44. Dukes JD, et al. Functional ESCRT machinery is required for constitutive recycling of claudin-1 and maintenance of polarity in vertebrate epithelial cells. Mol Biol Cell. 2011;22(17):3192-3205. 